\title{
TIGHTNESS AND MATERIAL ASPECTS OF BOLTED FLANGE CONNECTIONS WITH GASKETS OF NONLINEAR PROPERTIES EXPOSED TO VARIABLE LOADS
}

\begin{abstract}
The paper presents the problems regarding bolted flange connections with gaskets used in chemical, petrochemical and energy industry. The aim of the research is to present state of knowledge regarding pipelines and apparatus in industrial installations and rules and regulations regarding flange connections tightness. Additionally a calculation example regarding flange connection according to ASME VIII DIV 1 requirement and then detailed Finite Element Analysis presented; impact of nonlinear material properties (gasket loading unloading curves) on the connection tightness for complex loading programme is shown. It is finally concluded that in addition to usual design calculations more precise calculation is needed to fully verify behaviour of sealed connection at complex extreme variable loadings. Material aspect is very important at designing, testing, service and maintenance; taking it into consideration may avoid many problems related to safe exploitation.
\end{abstract}

Keywords: material, bolted flange connection, gasket, tightness

\section{Introduction}

There are hundreds of thousands of bolted-flange connections with gaskets, valves, pumps and compressors in chemical, petrochemical, refinery and energy industries. Such fittings in apparatus and pipelines are sources of media leakage. In chemical industry the operations are extended up to 5 years without overhauls. In energy industry industrial plants used to apply a continuous production methods, now there is necessity to switch to new operation conditions. They move from continuous to task production. Now installations must operate in changeable modes, they are expected to work in extreme conditions and might be instantly and unexpectedly stopped. Pipes and apparatus are heated and cooled in short periods and are vulnerable to material expansion and stress changes which leads to leaks. Furthermore, life time of steam turbine power systems in Poland which were designed for 200,000 working hours, is now extended to 300,000 or even to 350,000 hours. Old installations could easily failure and on the occasion of overhauls new parts made from new materials are used. It is necessary to address such kind of problems from many points of view. Based on detailed analysis adequate corrections are suggested. New designs must consider new constructions, new materials, requirements regarding tightness, existing and approaching rules and regulations and all of them are interconnected. Safe operation is then restored and capacity of the system improved remarkable.

The aim of the paper is to present state of knowledge regarding pipelines and various devices in industrial installations and rules and regulations regarding tightness. Additionally a calculation example regarding bolted flange connection(Fig 1) according to ASME VIII DIV 1 requirement and then detailed
Finite Element Analysis (FEA) is presented; impact of nonlinear material properties (gasket loading unloading curves) on the connection tightness for complex loading programme is shown. It is finally concluded that in addition to usual design calculations much more precise FEA is often needed to fully verify behaviour of sealed connection at complex extreme variable loadings. Material aspect is very important at designing, testing, service and maintenance; taking it into consideration may avoid many problems related to safe exploitation.

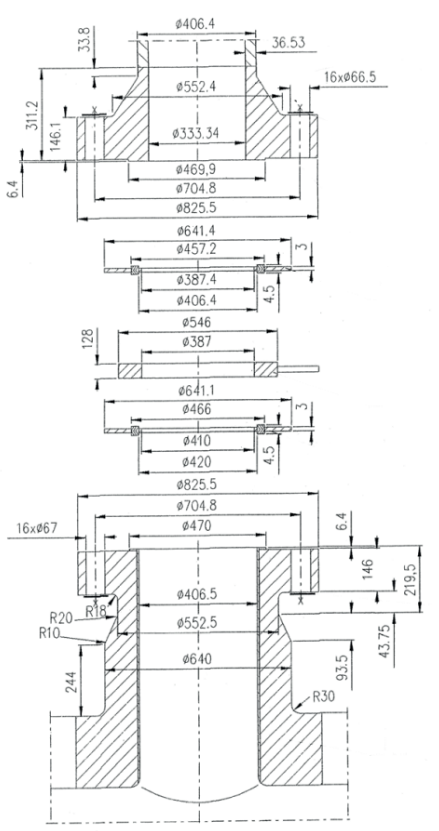

Fig. 1. Bolted flange connection of T4 nozzle

* WARSAW UNIVERSITY OF TECHNOLOGY, COLLEGE OF ECONOMICS AND SOCIAL SCIENCES, 17 LUKASIEWICZA STR. PLOCK, POLAND

** WARSAW UNIVERSITY OF TECHNOLOGY, FACULTY OF POWER AND AERONAUTICAL ENGINEERING, 24 NOWOWIEJSKA STR., 00-665 WARSAW, POLAND

*** WARSAW UNIVERSITY OF TECHNOLOGY, FACULTY OF MATERIALS SCIENCE AND ENGINEERING, 141 WOŁOSKA STR., 02-507 WARSAW, POLAND

\# Corresponding author: rpwalcza@pw.plock.pl 


\section{Theory}

Pipelines and technical apparatus are commonly used in industry. They are used for the processing and transfer of media in power engineering, heat engineering, and in chemical and petrochemical industries. They often operate in extreme technical conditions in terms of pressure and temperature of the medium being transferred, and technological processes (e.g. a change of the medium flow path in a system) [1].

At present, for economic reasons, there is a tendency in the industry to expand the time of operation of machinery, which causes that the length of the designed service life is overrun. This is related to a change in mechanical properties of the component parts that is affected by its operation history. In this case, an adequate selection of materials for the construction of such pipelines and apparatus is a particularly important question.

In order to select a material for the construction of such systems it is required to conduct an analysis of the working conditions, corrosive power of the environment and the medium being forced through as well as the assembling conditions. The application of a higher than the required steel grade increases the cost of the investment project. On the other hand, the use of steel grade of a too low quality decreases the degree of performance reliability and may be the cause of fault cracks and corrosive leakages occurring during their operation. Materials designed for pipelines and apparatus should have defined strength properties. The tensile strength value criteria $\mathrm{Rm}$ and the yield point Re may not be lower than the values adopted for strength calculations. Crack resistance during strength tests and under conditions of service load during the use are also important. Under assembling conditions, such material should have good welding properties in terms of its chemical composition as well as high corrosive resistance with relation to the operating medium.

The content of noxious components such as phosphorus or sulphur is limited to the $0.04 \%$ and $0.05 \%$ respectively. The content and mutual correlation of carbon and manganese is also limited since a high content of those elements hinders obtaining good weldability. If the aforementioned limitations are not observed, cracks may occur in the product particularly in the heat-affected area. With an increased content of carbon and manganese, it is extremely important to weld the joint penetration layer after heating the welded joint to an adequate temperature defined by the welding procedure and performance of a hot layer directly after completion of the joint penetration. Nitride and carbide forming (vanadium, niobium and titanium) alloy micro-additions in the steel not only increase the limit of the yield point and the limit of the tensile strength but unfortunately, they also limit weldability. Thus, there are certain thresholds that limit their application with respect to their content in the steel.

Welded joint defects represent a serious problem and are one of the most common causes of pipeline and apparatus failures. This is related to both discontinuities formed directly in the fusion welds and defects in the heat-affected areas. Large-diameter pipe welding process is found to be one of the most difficult aspects of steelwork assembly. The fusion welds must be performed in such a manner that the heat created in the process does not cause an alteration in the component part shape or the structure of the material. Furthermore, the welding process is conducted in all directions which may lead to melted metal flowing down in the effect of which the wall thickness in the fusion weld site is altered. A considerable thickness of the welded component parts causes an additional problem. The re-melting process has to cover the entire material because it is only then that the fusion weld ensures sufficient fusion weld strength. Additionally, the welding process of large diameter pipes is performed both from their exterior and the interior. Now new welding technologies may improve the quality of welds $[2,3]$.

Pipelines and apparatus, as any steelwork, underlie degradation as a result of corrosion processes [4]. Interior wall corrosion is most frequently associated with forcing hydrocarbons through. This is primarily hydrogen corrosion. Interior corrosion is also connected with medium pollution such as water. Since the materials that are in use include mainly low-alloy steels, the main corrosion type is uniform corrosion and pitting.

Wall erosion represents a serious hazard for materials used for pipelines and apparatus. It is caused mainly by the activity of the transported medium that, as it is in the case of petroleum, has much greater viscosity and inertia than water and includes a large number of impurities such as various types of solid particles. The problem concerns mainly pipe elbows and sites where pipe diameters are changed in which the flow direction and pressure of the medium is altered. Erosion initiation causes turbulent flow which significantly accelerates the erosion process. Erosion causes pipe wall thinning which results in the deterioration of mechanical properties.

The formation of hazardous fatigue defects may also be caused by another factor, i.e. stresses connected by changes of temperature and pressure of the medium as well as soil movements. Such stresses result, i.e. in formation and spreading of cracks as well as excessive strengthening of the material decreasing its elasticity/resilience [5].

At the apparatus designing stage and during their operation, pipe flange and bolted joints represent the crucial points that have a direct effect on steelwork integrity. The coefficient of linear expansion that is a material constant dependent on chemical composition and material structure has a prevailing influence on the characteristics of the fusion welds. As a result of joining two pipe flanges made from different steel types with the screws also made from different materials particular component parts are characterized by different stainless steel (SS) and carbon steel (CS) linear expansion dependent on the working temperature. Such system is particularly sensitive to dynamic temperature fluctuations that occur, for instance, during the starting and stopping the operation of the system. In extreme cases, an improper selection of materials for the operating conditions and servicing procedures may result in the destruction of the fusion weld and its loss of leaktightness. The system of a pipe flange fusion weld joined with components made from materials of differing linear expansion and badly chosen gasket, as well as, operational conditions such as variable temperature and pressure, and aggressive operating medium represents potentially the weakest point that causes the hazard of the loss of strength of the components welded together. 
Due to the tightness of the bolted flange connections the most important factor, next to the characteristics of the seals, is flexibility (stiffness) of the connection itself.

Especially dangerous are the states of rapid changes of connections temperature when in case of warming up the temperature of pipes and flanges connection is growing faster than the temperature of screws which sometimes causes significant increase in load and in this reason permanent gasket deformation.

After temperature equalization, when the temperature gradient decreases or during cooling the bolted flange connection can leak. When the materials of better mechanical properties are used it could be achieved better flexibility of the connections what leads to better tightness, especially in unstable conditions during fast start-up or stopping and in changing temperatures. This reduces the tendency to excessive compression of gaskets during connection overloading. Another method of reducing leakages is to use elements protecting gaskets from accidental crushing; those are centering rings or spring washers.

\subsection{Rules and regulations for bolted flange connections with gaskets}

Products of primary emissions (by-products of combustion, fumes from sewage etc.) and secondary emissions (fugitive emissions like volatile hydrocarbons, steam emitted from different types of seals, valves and tanks) are the direct cause of environmental pollution. Polish rules and regulations do not limit both types of emissions; they only govern their overall volume $[6,7]$ thus the industry is not interested in reducing the fugitive emission. The main sources of secondary emissions in the chemical and petrochemical industries are Bolted Flange Connections (BFCs), pumps, compressors, fittings, valves etc. where BFCs make up the substantial part of culprit facilities [8]. BFC must be equipped with gaskets in order to ensure leakage tightness, but zero emission is impossible. All BFCs constructions are provided with acceptable leaks according to environmental legislation [9], however achieving the required tightness depend on correct selection of constructions and materials of joints and gaskets.

In order to structure the fugitive emission the concept of tightness classes was introduced; they are closely related to leakage limits and coefficients to strength calculation of BFCs. The coefficients determine the size of bolds pre-load to ensure adequate pressure on the gasket and hence reduce leakages. In the European Union the tightness classes are defined by several standards. EN 13555 [10] and DIN 28090 [11] identify leakage test methods and 3 main tightness classes: $\mathrm{L}_{1,0}, \mathrm{~L}_{0,1}$ i $\mathrm{L}_{0,01}$ (respectively the leakage of $1.0 ; 0.1 ; 0.01 \mathrm{mg} /(\mathrm{m} \cdot \mathrm{s})$. Other standards like AD-M/DIN, WUDT, ASME refer to strength calculations, where required coefficients must be chosen from gaskets manufacturers catalogues. The vast majority of European petrochemical and chemical plants employ the $\mathrm{L}=0.01 \mathrm{mg} /(\mathrm{m} \cdot \mathrm{s})$; however in Germany the TA-Luft VDI 2440/ VDI 2200standard is applied where the maximum leakage of $10-4 \mathrm{mbar} \cdot 1 /(\mathrm{s} \cdot \mathrm{m})$ is specified.

In Polish chemical and petrochemical plants no rules and regulations regarding tightness classes have been applied. Vast majority of installations were designed and built in accordance with American codes ASME, where calculation coefficients meet $\mathrm{L} \sim 0.1 \mathrm{mg} /(\mathrm{m} \cdot \mathrm{s})$ tightness class. Assuming that over one million of bolted flange connections are used in Poland, raising the tightness class from $\mathrm{L}=0.1 \mathrm{mg} /(\mathrm{m} \cdot \mathrm{s})$ to $\mathrm{L}=0.01 \mathrm{mg} /(\mathrm{m} \cdot \mathrm{s})$ might significantly reduce leakage i.e. $3000 \mathrm{~kg}$ per year. It is possible to achieve better tightness using high quality gaskets of proper construction and flange connection parts made from correct materials.

The gasket is a relatively inexpensive component of the installation system. The leakage caused by gasket damage can cause the break in plants operation and result in additional costs such as additional labour costs and loss of production, which cannot be compared to the most expensive gasket.

Since 1.05.2004, in accordance with the PED 97-23 WE [12] and Directive 2001/95/EC [13], the only responsible for the equipment are persons (producers or distributors) who place it on the Community market, hence they are responsible for the technical documentation and all components including BFCs and gaskets. For the equipment used at the plant the user takes over the responsibility.

In the European Union recognized institutes and research laboratories i.e. the Münster University of Applied Science, ASE Ltd. Cambridge UK, CETIM Nantes France, IRC Petten Nederlands, AMTEC Lauffen Germany, MPA University of Stuttgart Germany are responsible for both verification of data presented in catalogues and the quality of the seals. Products recognized by the Institutes as correct are placed on the websitewww.gasketdata.org.

In chemical plants the proper gaskets and high quality materials should be installed. In bolted flange connections many types of gaskets are used, i.e.: non-metallic soft gaskets, serrated gaskets, spiral wound gaskets, corrugated metal gaskets, metal gaskets of ring-type-joint, lens etc. type, graphite gaskets, bare and jacketed. The gaskets must ensure chemical resistance, fire resistance, the appropriate tightness using minimal load, the possibility to use at high pressures (in cold and hot conditions), high temperature resistance, resistance to aging, preservation of elasticity at varying loads, blowout resistance, ability to compensate material inequalities, resistance to relaxation in long time period - minimum 5 years, good exploitation service.

Formal requirements in the European Union for highquality gaskets include permissions pursuant to standards TALuft [14], Fire Safe acc. to API 607/BS 6755, BAM, DVGW and others.

\subsection{Exploitation of bolted flange connections with gaskets}

Replacing the seals or other components of the connection often requires stopping the entire system for a few days, what with the daily production of up to several million dollars gave big losses for the enterprises and pose a threat to the environment.

ASME-B16.5 [15] standard does not include additional loads and in the apparatus designs they are not always taken into account. These codes advise tighten the screws on the start-up in the hot operating conditions. In practice it is not possible for large installations and impossible for connections inside apparatus. 
DIN 2505 [16] code introduces additional calculation coefficients of 0.1 and 0.2 that increase design bolds loads. Other guides assume additional coefficients to loads depending on the modulus of elasticity of the bolts (Eq. 1):

$$
\frac{E_{t 20^{o} C}}{E_{t}}
$$

and the additional loads increasing the design pressure, acc.to the formula (Eq. 2)

$$
P_{\mathrm{ad}}=\frac{4 \mathrm{~F}}{\pi \mathrm{D}_{0}^{2}}+\frac{16 \mathrm{M}}{\pi \mathrm{D}_{0}^{3}}
$$

where:

$\mathrm{F}$ - axial force acting on the flange connection, $\mathrm{N}$,

$\mathrm{P}_{\mathrm{ad}}$ - additional pressure added to designed pressure, $\mathrm{MPa}$,

$\mathrm{D}_{0}$ - average gasket diameter, $\mathrm{mm}$,

$\mathrm{M}$ - the square root of the sum of the moments acting on the flange connection, $\mathrm{MPa}$,

$E_{t^{-}}$modulus of elasticity (Young modulus) in designed temperature, $\mathrm{N} / \mathrm{m}^{2}$,

$E_{t 20^{\circ} C}$ modulus of elasticity in $20^{\circ} \mathrm{C}, \mathrm{N} / \mathrm{m}^{2}$.

\section{Example calculation analysis and calculation of bolted flange connection, which suffered a major accident on responsible petrochemical plant}

In the hydrogen plants hydrogen leakage at flange connection of nozzle T4 (Fig. 1, Fig. 2) of pipeline on hydrogen generation division occurred after several startups. Interim repair was made by external band and injection of sealing material between flanges. Unfortunately some residual outflow of hydrogen was still observed at heads of bolts which implied that gas migrated along bolts. It was suggested to apply unique washers replacing previous bellow bolt heads to plug connection. This solution required to reassemble all bolts sequentially one by one in operating conditions. It was necessary to explain reasons for the leakage observed and to analyze process of washers exchange considering normal pressure distribution on gaskets and stress distribution on flange connection elements exposed to operation extreme load conditions. Calculations according to ASME and DIN standards and finite element method modelling served to achieve this objective.
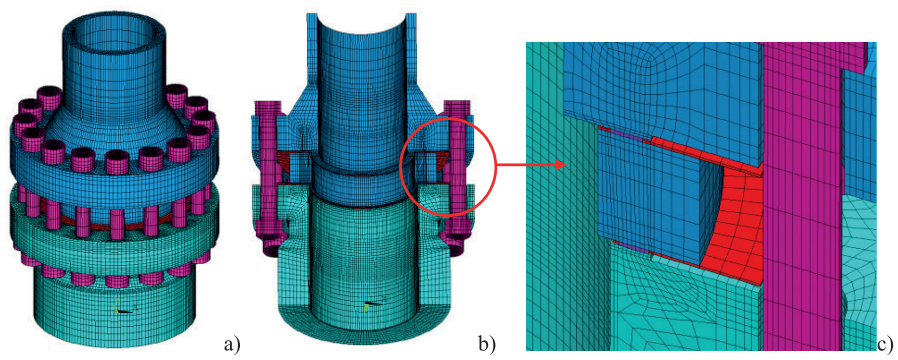

Fig. 2. Finite element model of flange connection at T4 nozzle of heat exchanger E-04 on in petrochemical plant: full view a), half view b), distancing ring with two graphite-ss spiralwound gaskets and internal and external protective rings
Common steel material properties were used in calculation:
- Young modulus

- Poisson ratio

- mass density

- thermal expansion coefficient

- thermal conductivity

- specific heat
$\mathrm{E}=2.0 \mathrm{E}+11 \mathrm{~N} / \mathrm{m}^{2}$ for $\mathrm{t}=20-100^{\circ} \mathrm{C}$, $\mathrm{v}=0.3$, $\rho=7.850 \mathrm{E}+3 \mathrm{~kg} / \mathrm{m}^{3}$, $\beta=1.2 \mathrm{E}-5 \mathrm{1} / \mathrm{mK}$, $\lambda=46.7 \mathrm{~W} / \mathrm{mK}$, $\mathrm{c}=444 \mathrm{~J} / \mathrm{kgK}$,
Flange was made of steel A 336 F22 cl.3, counterflange of steel A 182 F11 cl.2 and SA 193 Gr.B7 was used for bolts. Elasto-perfectly plastic material model (no hardening) was adopted for these materials assuming following yield stress values at elevated temperature $\mathrm{T}=268^{\circ} \mathrm{C}: \mathrm{Re}=248 \mathrm{MPa}$ for flange, $\mathrm{Re}=223 \mathrm{MPa}$ for counterflange and $\mathrm{Re}=550 \mathrm{MPa}$ for bolts.

Two gaskets separated by distancing ring are of graphitess spiral wound type with two protective rings (internal and external)each. The minimum required gasket pressure equals to $69 \mathrm{MPa}$ (for ASME regulations) and $55 \mathrm{MPa}$ (for DIN reg.) and the maximum ranges from $300 \mathrm{MPa}$ for room temp. to $250 \mathrm{MPa}$ for $\mathrm{T}=250^{\circ} \mathrm{C}$ according to IDT (Germany) data. The nonlinear stiffening gasket loading curve and linear unloadings of different slope (depending on load level) assumed for analysis (Fig.3).

Flange connection 16" in nozzle T4 of heat exchanger E-04 (Fig. 1) was originally calculated without taking into account external loads from pipelines. The design parameters and material data of the connection are shown in TABLE 1.There were conducted new calculations according to ASME VIII DIV. 1 considering additional load according to Eq. (2).

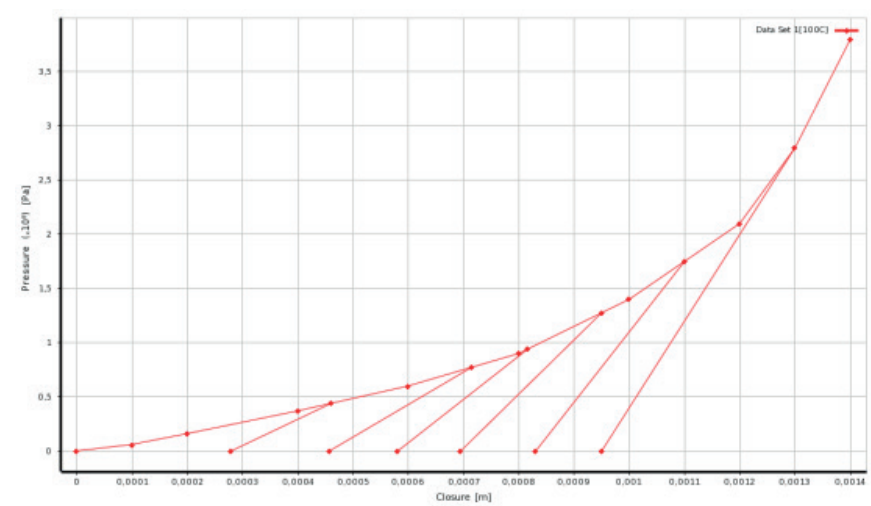

Fig. 3. Compression - unloading curves for graphite-ss spiral wound gasket model.

Analysis of stress in the flange showed that the tangential stress in the flange $\mathrm{Sf}=161.7 \mathrm{MPa}$ were greater than acceptable stress $\mathrm{Sfa}=129.66 \mathrm{MPa}$. These results were obtained during the calculation compliant with the aforesaid standard. In addition, stress in the flange were tested according to DIN 2505. Stress in the collar neck during operating conditions were equal to $71.9 \mathrm{MPa}$ and were smaller than acceptable $165 \mathrm{MPa}$. According to calculation results flange stress did not exceed the standard quantities and the connection should meet tightness requirements. Additionally, to confirm that conclusion, a detailed calculations using FEM method were conducted. 
Force and moment of force reactions of pipeline on the flange connection were transformed to equivalent internal pressure increase according to formulas (Eq. 1, 2). Than both the structure as well as all the loads might be treated as cyclically symmetric which helps to reduce dimension of finite element computational model to the single sector (Fig.2). Eight node 3D elasto-plastic elements were used for metal structure, particular nonlinear elements to represent gasket and usual frictional contact elements on contact surfaces.

Extreme loading conditions including temperature, internal pressure bolt pretension and external loads are strongly influencing flange connection to remain tight. Both absolute values of listed loadings as well as their gradients and time runs should be taken into account when analysing structure behavior. Original design calculation check had been done for steady state conditions and internal design pressure $\operatorname{Pr}=14.4 \mathrm{MPa}$ (without taking into account quite big pipeline force and moment of force reactions). In the present FEA approach of T4 nozzle flange connection following load interactions were assumed in a form of loading program:

1. Assembly state represented by bolt pretension of 16 bolts of $0.364 \mathrm{MN}$ per bolt which results of total force $\mathrm{N}=5.82 \mathrm{MN}$.

2. Design condition "cold": bolt pretension locked (by initial strain) + internal pressure (incl. force \& moment of force pipeline reactions) $\mathrm{Ptr}=\mathrm{Pr}+\mathrm{Peq}=14.4+6.82=21.22 \mathrm{MPa}+$ uniform temperature.

3. Heating up state (design condition): bolt pretension locked (by initial strain) + internal pressure $\mathrm{Ptr}=21.22 \mathrm{MPa}+$ temperature of bolts lower by $\Delta \mathrm{T}=268 \mathrm{OC}$ than flanges.

4. Operation state "hot" design condition: bolt pretension locked (by initial strain) + internal pressure Ptr= 21.22MPa+uniform elevated temperature of bolts and flanges.

5. Service state: removing of single bolt in operation state (acc. to point 4)

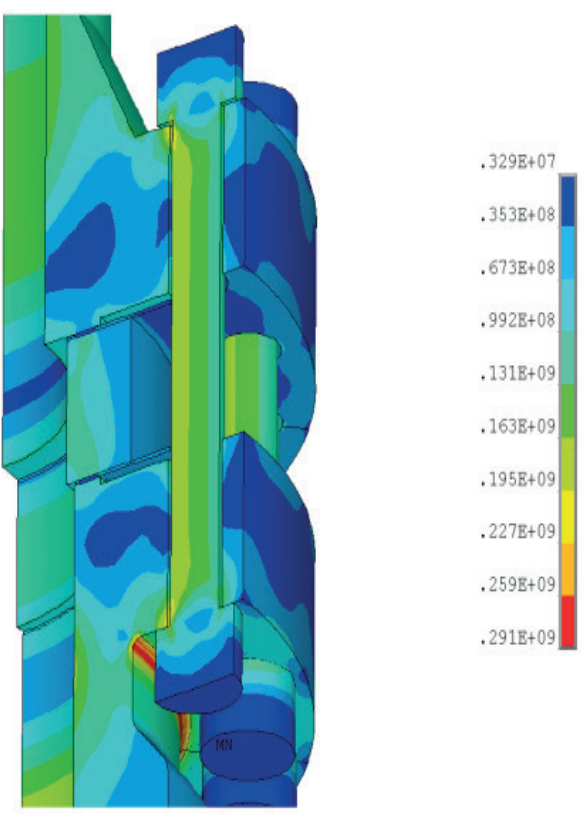

Fig. 4. Equivalent Huber-Mises stress distribution [Pa] on flange connection at $\mathrm{T} 4$ nozzle of heat exchanger E-04 for design pressure $\mathrm{P}=21.22 \mathrm{MPa}$ and uniform temperature

\section{Results}

The maximum normal gasket pressure for assembly state (total bolt pretension $\mathrm{N}=5.82 \mathrm{MN}$ ) reach $\sigma=193 \mathrm{MPa}$ on flange gasket and $\sigma=180 \mathrm{MPa}$ on counterflange gasket (Fig.6a) and drops to $\sigma=104-120 \mathrm{MPa}$ and $\sigma=110-124 \mathrm{MPa}$ under internal pressure $\mathrm{P}=21.22 \mathrm{MPa}$ (Fig.6b). Protection rings remain inactive since the maximum gasket closure is about $1 \mathrm{~mm}$ compared to $1.5 \mathrm{~mm}$ needed to rings would get in contact with flanges. The maximum equivalent Huber-Mises stress appear on the flange neck and equals to $\sigma^{\mathrm{HM}}=291 \mathrm{MPa}$ (Fig.4). While fast heating up when the difference of temperatures between flanges and bolts reach $\Delta \mathrm{T}=268^{\circ} \mathrm{C}$ normal gasket pressure rise up to $\sigma=246$ -

TABLE 1

Material data for heat exchanger cover flange and bolts

\begin{tabular}{|c|c|c|c|}
\hline \hline Material data & Flange & Bolts & Design data \\
\hline Material type & A 366 F22 Cl.3 & SA 193 B7 Table 3 & Pressure P=21.22 MPa \\
\hline Standard & ASME Tab. 1.A & ASME Tab. 1.A & Temperature $\mathrm{t}_{\mathrm{o}}=268^{\circ} \mathrm{C}$ \\
\hline $\begin{array}{c}\text { Allowable stress in } 20^{\circ} \mathrm{C} \\
\text { Acc. to ASME VIII DIV. } 1\end{array}$ & $\mathrm{~S}_{\mathrm{fa}}=129.66 \mathrm{MPa}$ & $\mathrm{S}_{\mathrm{a}}=158.62 \mathrm{MPa}$ & \\
\hline $\begin{array}{c}\text { Allowable stress in design temperature } \\
\text { acc. to ASME VIII DIV. } 1\end{array}$ & $\mathrm{~S}_{\mathrm{fo}}=129.6 \mathrm{MPa}$ & $\mathrm{S}_{\mathrm{b}}=158.62 \mathrm{MPa}$ & \\
\hline $\begin{array}{c}\text { Allowable stress in } 20^{\circ} \mathrm{C} \\
\text { acc. to DIN } 2505\end{array}$ & $282.09 \mathrm{MPa}$ & $545.83 \mathrm{MPa}$ & \\
\hline $\begin{array}{c}\text { Allowable stress in design temperature } \\
\text { acc. to DIN } 2505\end{array}$ & $165 \mathrm{MPa}$ & $343.94 \mathrm{MPa}$ & \\
\hline
\end{tabular}


$284 \mathrm{MPa}$ on flange gasket and $\sigma=295-256 \mathrm{MPa}$ on counterflange reaching the maximum value on the external edge (Fig.6c).
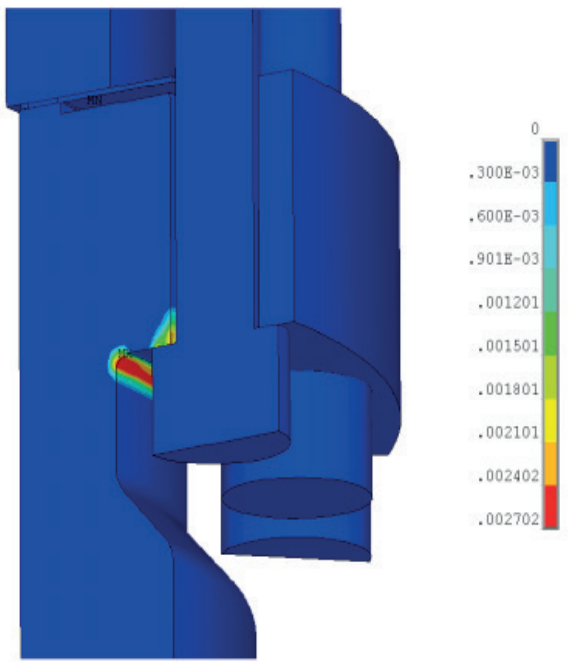

Fig. 5. Equivalent plastic strain [-] on flange connection at T4 nozzle of heat exchanger $\mathrm{E}-04$ for design pressure $\mathrm{P}=21.22 \mathrm{MPa}$ and heating up condition when flanges are $\Delta \mathrm{T}=268^{\circ} \mathrm{C}$ warmer than bolts

Nevertheless there is still no contact between protective rings and flanges but opening reduces to $0.2 \mathrm{~mm}$. Thisextreme loading case results of plastic flange deformation which occur on the neck and near bolt's head (Fig.5). However maximum equivalent plastic strain remain relatively small $\mathrm{epl}=2.7 \%$. At equal temperatures for "hot" operating state normal gasket pressures drops to only just $\sigma=53-75 \mathrm{MPa}$ on flange gasket and $\sigma=53-77 \mathrm{MPa}$ on counterflange gasket (Fig.6d) which is at the minimum sealing value and suggests possible leakage. What is worse gasket pressure is lower on the external edge. However external protective ring still preserve the gasket not to blow out. Removing a single bolt at steady state operating condition results in further normal gasket pressure reduction to $\sigma=28-59 \mathrm{MPa}$ which is below minimum sealing value (Fig. 7b) and imply possible outflow increase. External ring should still keep gasket in its place to follow scenario "leak but not burst". Obtained equivalent stress distribution (Fig.7a) ensures that washer replacement is a safe procedure.

\section{Discussion}

Based on engineering calculations and detailed FEM analyses of strain, stress, gasket closure and pressure distributions carried out for extreme design loads and their interactions it may be concluded as following:

1. T4 nozzle flange connection remain tight under design loads for steady state conditions $(\mathrm{N}=5.82 \mathrm{MN}$, $\mathrm{P}_{\mathrm{r}}=14.4 \mathrm{MPa}, \mathrm{T}=268^{\circ} \mathrm{C}$ ) even if the extreme force and moment of force pipeline reactions are taken to increase design pressure up to $\mathrm{P}_{\mathrm{tr}}=\mathrm{P}_{\mathrm{r}}+\mathrm{P}_{\mathrm{eq}}=14.4+6.82=21.22 \mathrm{MPa}$.

2. Extreme temperature difference between flanges and bolts while heating up the pipeline results in remarkable gasket pressure increase up to maximum allowable values. In the following uniform temperature "hot" state (for sustained pipeline reaction $\mathrm{P}_{\mathrm{tr}}=21.22 \mathrm{MPa}$ ) gasket normal pressure drops down to $\sigma=53-75 \mathrm{MPa}$ which is the minimum sealing value and indicates possible leak.

3. Protective gasket rings are not in contact while nominal and extreme load condition. However the external ring preserve the gasket not to blow out. Leak but not burst occur.

4. Average stress at flange connection is on moderate level and remain below allowable design value for all load cases analyzed. Despite of that there are several stress concentration regions like flange neck and hole for bolt. Plastic deformation appear there at extreme heating up but it is local and does not exceed $\varepsilon_{\mathrm{pl}}=2.7 \%$.

5. Exchanging washers to plug flange connection can be

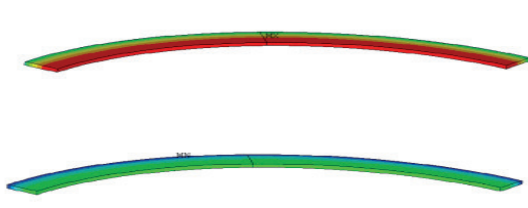

a)

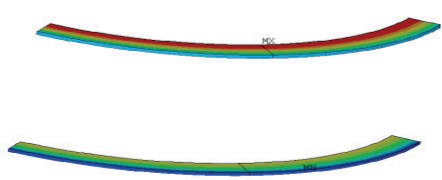

c)
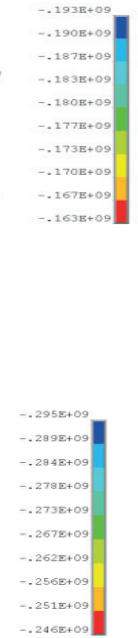

b)

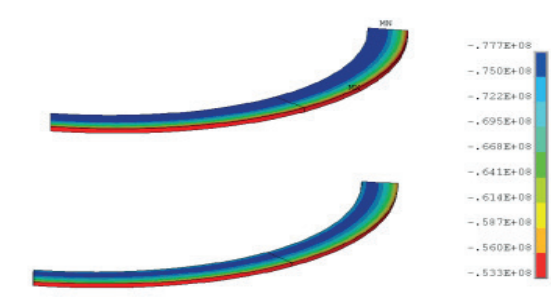

d)

Fig. 6. Normal gasket pressure $[\mathrm{Pa}]$ on flange connection gaskets at $\mathrm{T} 4$ nozzle of heat exchanger $\mathrm{E}-04$ for: bolt pretension $\mathrm{N}=364 \mathrm{kN}$ at assembly a), under design pressure $\mathrm{P}=21.22 \mathrm{MPa}$ and uniform temeprature $\mathrm{b}$ ), while fast heating up when flanges are $\Delta \mathrm{T}=268 \mathrm{OCwarmer}$ than bolts c) and for steady state at design pressure and temperature d) 


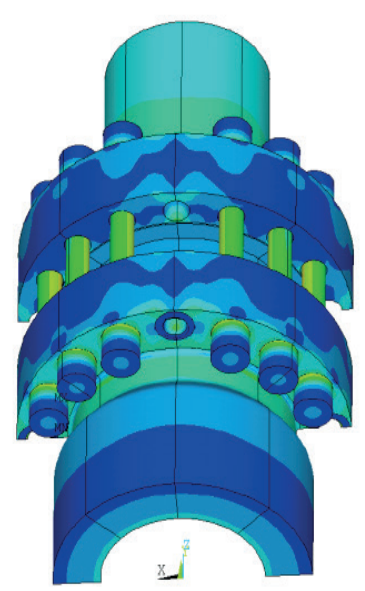

a)
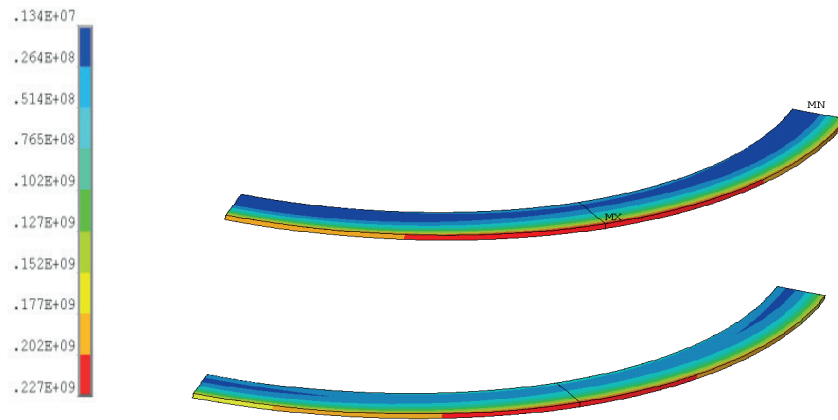

b)

Fig.7. Flange connection at T4 nozzle of heat exchanger E-04 after remooving single bolt in steady state design condition: Huber-Mises Equivalent stress distribution $[\mathrm{Pa}] \mathrm{a})$, normal gasket pressure $[\mathrm{Pa}] \mathrm{b}$ )

done but temporary hydrogen outflow increase has to be taken into account.

Summarizing it is worth to emphasize that the most apparent reason for hydrogen leakage is interaction of pipeline reaction and extreme difference between temperatures of flange and bolts. According to assumed gasket loading unloading curves protective rings should be slightly thicker (by $0.2-$ $0.3 \mathrm{~mm}$ ) in order to secure gasket material against overloading. On the other hand use of modified gasket of lower subsidence and more elastic relief (unloading) for analyzed connection would be strongly preferred.

\section{Conclusion}

In order to preserve harmless and clean operations of Polish energy, chemical, refinery and petrochemical systems which help to protect our environment following recommendations should be taken into account:

- apply high quality materials for flange connection structural elements and gaskets approved by international or national regulations and research institutes,

- require to design flange connections, use appropriate materials and computational and verification procedures which preserve stable, tight and safe operation for extreme and variable loads keeping at least 5 years long period before maintenance or servicing,

- include modern analysis tools such as Finite Element Analysis at the design level for responsible connection working in complex variable load conditions to verify any possible load interactions,

- $\quad$ establish categories of sealing requirements for particular application fields in a form of national or trade regulations for design and maintenance purposes,

- $\quad$ evolve review and monitoring system for leakage and bolt pretension measurement or indication, particular bolt pretension set up and verification procedures should be established for flammable and explosive media,
- $\quad$ map out assembly, operation and maintenance instructions for different flange connection categories, promote trainings for operation and maintenance services for mantle/dismantle works.

Design of flange connection and gasket system for complex and high parameters or dangerous systems should be verified by detailed analysis and testing before approval.

\section{REFERENCES}

[1] P. Fassinaa, F.Bolzonib, G.Fumagallib, L.Lazzarib, L.Verganic, A.Sciuccatic, Influence of Hydrogen and Low Temperature on Pipeline Steels Mechanical Behaviour, Procedia Engineering 10, 3226-3234 (2011).

[2] D. Hadryś, T. Węgrzyn, J. Piwnik, The Effect of Different Micro-Jet Streams Number on Plastic Properties of Welds, Archives of Metallurgy and Materials 60, 3A (2015).

[3] T. Węgrzyn, J. Piwnik, Ł. Wszołek, W. Tarasiuk, Shaft Wear after Surfacing with Micro-Jet Cooling, Archives of Metallurgy and Materials 60, 4 (2015).

[4] J.G. Speight, Corrosion Monitoring and Control, Oil and Gas Corrosion Prevention, From Surface Facilities to Refineries 109-149 (2014).

[5] H.M. Nykyforchyn, K.J. Kurzydlowski, E. Lunarska, Hydrogen degradation of steels under long-term in-service conditions, Environment-Induced Cracking of Materials, Volume 2: Prediction, Industrial Developments and Evaluation 349-361 (2008).

[6] Directive 2010/75/EU of the European Parliament and of the Council of 24 November 2010 on industrial emissions (integrated pollution prevention and control).

[7] Prawo ochrony środowiska. Ustawa z dnia 27 kwietnia $2001 \mathrm{r}$. Dz.U. 2001 nr 62 poz. 627.

[8] FSA/ESA Guidelines for Safe Seal Usage - Flanges \& Gaskets, Part 1. ESA/FSA Publication No. 009/98, (1998). http://europeansealing.com/uploads/resources/publications/ ESA-FSA-Guidelines-Flange-Gaskets-009_98_ENG.pdf

[9] D. Bathen, C. Hummelt, J. Meisel: Emissions on Flanged 
Joints, Vulkan Verlag, Essen. (2000).

[10] EN 13445 Unfired Pressure Vessels Standard.

[11] DIN 28090 Static Gaskets for Flange Connections Standard.

[12] Pressure Equipment Directive (PED) Directive 97-23 WE of the European Parliament and of the Council of 29 May 1997 on the approximation of the laws of the Member States concerning pressure equipment. http://eur-lex.europa.eu/ legal-content/EN/TXT/PDF/?uri=CELEX:01997L0023- 20130101\&from $=\mathrm{EN}$

[13] Directive 2001/95/EC of the European Parliament and of the council of 3 December 2001 on general product safety.

[14] VDI 2440: Reducing Emissions from Mineral Oil Refineries. Beuth Verlag, Berlin, 2000.

[15] ASME-B16.5-2003. Pipe Flanges and Flanged Fittings.

[16] DIN V 2505 Calculation of flanged joints. 